\title{
Efecto de un proceso grupal de meditación sobre el estrés percibido y las estrategias de afrontamiento en un contexto carcelario
}

\section{Effect of a Group Meditation Process on Perceived Stress and Coping Strategies in a Prison Context}

\author{
María Nazareth Mora-Arias, \\ Lizbeth Solano-Alpízar, Bradly Marín-Picado, \\ Jorge Esteban Prado-Calderón \\ Universidad de Costa Rica, Costa Rica
}

\begin{abstract}
Resumen
La privación de libertad es un suceso que impacta los niveles de estrés y requiere estrategias de afrontamiento. En la cárcel Jorge Arturo Montero Castro de Costa Rica se implementó un proceso grupal de meditación con personas recluidas para ayudarles a sobrellevar el encarcelamiento. Para aportar evidencia científica sobre la efectividad de esta intervención, se realizó un estudio mixto de triangulación con modelo de convergencia. Cuantitativamente, se evaluó si la intervención generó cambios en los niveles de estrés percibido de los participantes. El diseño fue experimental con preprueba-posprueba y grupo control de lista de espera. La muestra fue de 32 hombres (16 en cada grupo). Cualitativamente, se exploró cómo influyó la intervención en los estilos de afrontamiento dentro del ambiente carcelario. El diseño fue un estudio de casos múltiples con muestreo intencional y se realizó una entrevista semiestructurada a cada participante del grupo de tratamiento. Además, se revisaron las consignas, los contenidos y el diseño de la intervención para evaluar su idoneidad. Los resultados cuantitativos no evidenciaron diferencias entre el grupo experimental y el grupo control; sin embargo, sobresalió la diferencia significativa en la subescala cognitiva $\left(F_{(1,15)}=5.01, p=.04\right)$ entre la preprueba y la posprueba del grupo de tratamiento. En los resultados cualitativos, los participantes expresaron que con el proceso lograron utilizar estrategias como autocontrol, reevaluación positiva y distanciamiento. Sobre el diseño de la intervención, sobresalen las fortalezas; pero, se considera que la intervención puede rediseñarse al incorporar los aspectos por mejorar para maximizar los beneficios.
\end{abstract}

Palabras clave: privación de libertad, meditación, Psicología de la salud, ambiente, estrés, afrontamiento

María Nazareth Mora-Arias, Lízbeth Solano-Alpízar, Bradly Marín-Picado y Jorge Esteban Prado-Calderón; Universidad de Costa Rica, Costa Rica.

La correspondencia en relación con este artículo se dirige a María Nazareth Mora-Arias, investigadora del Programa de Posgrado en Especialidades Médicas, Universidad de Costa Rica, Costa Rica. Dirección electrónica: marianazarethmoraarias@gmail.com 


\begin{abstract}
:
Because privation of liberty in jail affects health, a group meditation process with prisoners was implemented at the Jorge Arturo Montero Castro Institutional Care Center, in Costa Rica, to help cope with imprisonment. Due to gaps in the scientific approach to meditation effectiveness with prisoners in Costa Rica, a mixed method using convergence design and triangulation was carried out. A quantitative evaluation was made to ascertain if the meditation process generated changes in participant stress levels. A pretest-posttest experimental design with a wait-list control group was used. The sample was 32 men (16 in each group). A qualitative evaluation was made to explore how the intervention influenced coping in participants. Multiple case study design was used. Intentional sampling was performed, which resulted in working with 16 men of the meditation process group. Additionally, instructions, contents and design of the intervention were reviewed. Quantitatively, there were no differences between experimental group and control group on stress levels after the intervention; however, in the experimental group there was a significant difference in the cognitive subscale $\left(F_{(1,15)}=5.01, p=.04\right)$. Qualitatively, participants expressed that they began to use strategies like self-control, positive reevaluation and taking distance from the situation. Finally, strengths were recognized in the intervention, but also that there was room for improvement in order to maximize benefits.
\end{abstract}

Keywords: Imprisonment, Meditation, Health Psychology, Environment, Stress, Coping

Lazarus y Folkman (1986) definen el estrés psicológico como "una relación particular entre el individuo y el entorno, que es evaluado por éste como amenazante o desbordante de sus recursos y que pone en peligro su bienestar" (p. 43). En términos generales, el estrés es un proceso adaptativo en el que interactúan los estímulos ambientales; la evaluación cognitiva; las formas de afrontamiento; las respuestas neurovegetativas, motoras y cognitivo-conductuales y los factores moduladores personales del sujeto (De Camargo, 2010; Moser, 2014). Los estímulos del entorno no son en sí mismos estresantes, por lo que la reacción suscitada varía de acuerdo con la percepción del individuo (Cozzo \& Reich, 2016).

En este sentido, como resultado de la evaluación cognitiva de los estímulos ambientales, el individuo puede manifestar reacciones a nivel neurovegetativo (activación del sistema nervioso autónomo que genera cambios en el ritmo cardiaco, en la salivación, en funciones estomacales, sudoración, etc.), motor (sensaciones de temblor, dolor, paralización, debilidad, etc.) y cognitivo (dificultades en procesos de atención, memoria, etc.) (Cólica, 2015; De Camargo, 2010).

Como tal, el estrés no es negativo en el sentido estricto de la palabra; es la duración y la frecuencia excesivas de la respuesta natural de estrés la que representa un riesgo para el bienestar (Ramos, 2017; Ugarte, 2010). Así, se debe diferenciar el estrés agudo del estrés crónico. El primero es positivo al posibilitar la adaptación a una situación desconocida; en el segundo, la reacción de alarma se extiende en el tiempo, porque el estímulo estresante persiste en el entorno, no se elimina por incapacidad para enfrentarlo o, aunque ya no está presente, los sistemas internos o las interpretaciones de la persona siguen actuando como si lo estuviera (Sánchez, 2010).

En este escenario, la valoración cognitiva es mediadora entre los estímulos y las reacciones de estrés; por tanto, determina el carácter amenazante o no de los elementos del entorno y la manera de afrontarlos. En cuanto al afrontamiento, Lazarus y Folkman (1986) indican que las personas pueden 
EFECTO DE UN PROCESO GRUPAL DE MEDITACIÓN SOBRE EL ESTRÉS Y LAS ESTRATEGIAS DE AFRONTAMIENTO

hacer frente a las situaciones de estrés al distanciarse para no pensar en ellas y evitar que les afecte (distanciamiento); al recurrir a conductas como usar drogas, tomar medicamentos, etc. (huida-evitación); al buscar consejos, asesoramiento, comprensión (búsqueda de apoyo social); al tomar acciones directas para alterar las situaciones (confrontación); al reconocer el propio papel en la situación (aceptación de la responsabilidad); al realizar esfuerzos analíticos centrados en la situación con el fin de resolverla (planificación); al dotar a la situación de un significado positivo para el desarrollo personal (reevaluación positiva) y al procurar la regulación de las emociones y acciones (autocontrol). Con el fin de que las personas utilicen estrategias de afrontamiento que les beneficien y disminuya la respuesta de estrés crónico, la Psicología de la salud y ambiental buscan la transformación de la persona desde el interior-emociones y pensamientos- hacia el exterior -conductas- (Goleman, 2003; Harrison, 2004; Salom, 2005; Weiss, 2007).

A partir de las definiciones anteriores, se recalcan dos elementos importantes. En primer lugar, las personas pueden responder de manera diferente a una misma situación conforme a su personalidad e historia; en segundo lugar, la relación entre el individuo y el entorno puede modificarse en la medida en la que el sujeto cambie su valoración, aprenda a reducir la activación de sus sistemas internos y recurra a estrategias diferentes de afrontamiento.

En el caso específico de la privación de libertad, esta puede ser considerada como una experiencia altamente estresante (García-Jarillo, Caravaca-Sánchez, Sánchez-Alcaraz \& Luna, 2016). El encarcelamiento, además de restringir la libertad, obstaculiza la satisfacción de necesidades y el cumplimiento de metas. Además, conlleva el distanciamiento de las redes de apoyo (García-Jarillo et al., 2016).

Adicionalmente, los centros penitenciarios presentan una serie de condiciones que pueden ser fuentes de estrés como percepción de calor y ruido, olores desagradables, hacinamiento, falta de intimidad y conflictos "territoriales". Dichas situaciones desequilibran el organismo por ser estímulos ambientales valorados como peligrosos y demandantes (González \& Escobar, 2002).

En ese contexto, específicamente en el Ámbito C del Centro de Atención Institucional Jorge Arturo Montero Castro de Costa Rica (en adelante CAI JAMC), la psicóloga Kenya Lobo y el doctor Ignacio Salom elaboraron una intervención centrada en meditación con el fin de promover bienestar en las personas recluidas y ayudarles a sobrellevar la situación de encarcelamiento. Dicha intervención la han implementado durante aproximadamente 15 años.

Pese al tiempo de ejecución, no se había realizado un estudio empírico y sistemático sobre la efectividad de la intervención. Al respecto, cabe señalar que efectuar estudios con población penal implica realizar trámites diversos para resguardar la seguridad y enfrentar situaciones carcelarias (rotación de la población, cancelación de sesiones por requisas, etc.). Esos eventos dificultan la investigación y probablemente son parte de las razones por las que en Costa Rica hay poca documentación sobre la efectividad de intervenciones dirigidas a personas recluidas.

En línea con lo anterior, es relevante destacar que, previo a este estudio, en el país no se había efectuado investigación sobre el tema con la población meta. Esto constituye un problema, porque los excluye de los beneficios que les podría aportar la investigación: conocimiento, mejoramiento, reforzamiento y extensión de programas. Por ello, al reconocer la complejidad de la privación de libertad y 
la necesidad de contar con insumos sobre la efectividad de las intervenciones dirigidas a las personas recluidas del país, se decidió acoger la solicitud de las personas facilitadoras y realizar esta investigación.

Así, para dar cuenta de los alcances de la intervención, se estudió la experiencia del grupo de hombres privados de libertad, que participó en el proceso grupal de meditación de octubre del 2017 a febrero del 2018. El objetivo fue evaluar si el proceso grupal de meditación generó cambios en los niveles de estrés percibido de los participantes y explorar cómo influyó el proceso grupal de meditación en los estilos de afrontamiento de los usuarios dentro de un entorno carcelario. Además, dado el limitado conocimiento que existía en el país sobre este tipo de intervención con población penal, se incluyó como objetivo externo realizar una valoración al diseño, contenidos y consignas del proceso grupal de meditación para evaluar su idoneidad.

El equipo investigador se focalizó en el estrés percibido, porque en varios estudios se reporta que las intervenciones con meditación disminuyen los niveles de estrés en hombres privados de libertad (Barnert, Himelstein, Herbert, García-Romeu \& Chamberlain, 2014; Le \& Proulx, 2015; Nidich et al., 2016). Además, esta temática se consideró importante, porque el estrés es un problema que aqueja a la población de Costa Rica (Martínez, 2014) y probablemente esté presente en la población carcelaria (Mecanismo Nacional de Prevención de la Tortura, 2018). Asimismo, se contemplaron las estrategias de afrontamiento, dado que en estudios sobre meditación con personas recluidas se reportó que la práctica contribuyó a la adquisición de estrategias nuevas (Barnert et al., 2014; Pham, 2013; Ronel, Frid \& Timor, 2013). También, se contemplaron los estímulos ambientales que influyen en la experiencia negativamente, entendidos como estresores físicos (temperatura, olor y ruido) y psicosociales (territorialidad, hacinamiento, falta de intimidad, monotonía ambiental y falta de control sobre el entorno).

Para ello, se utilizó un diseño mixto de triangulación con modelo de convergencia. En este sentido, la revisión de investigaciones sobre meditación con personas privadas de libertad (Barnert, et al., 2014; Le \& Proulx, 2015; Nidich et al., 2016; Orme-Johnson \& Moore, 2003; Perelman et al., 2012; Pham, 2013; Rainforth, Alexander \& Cavanaugh, 2003; Ronel et al., 2013; Vannoy \& Hoyt, 2004) evidencian la pertinencia de evaluar los aportes de la meditación desde abordajes cuantitativos para destacar sus beneficios en el manejo del estrés, autocontrol, inteligencia emocional, adaptabilidad, disminución de la reincidencia, entre otros. Asimismo, los abordajes con enfoque cualitativo permitieron la captación del fenómeno desde las percepciones de los sujetos. Sobresalieron la importancia del trabajo grupal colaborativo y la actitud empática por parte de los facilitadores para promover el bienestar. Sin embargo, a diferencia de la presente investigación, pocos estudios contemplaron las condiciones ambientales de los centros penitenciarios para explicar los resultados, en tanto obviaron que, en esos entornos, las personas suelen afrontar situaciones desconocidas y cambios extremos.

\section{Método}

Para evaluar la efectividad del programa de meditación en la reducción de los niveles de estrés percibido y explorar los posibles cambios en los estilos de afrontamiento ante los estresores del contexto, en esta investigación se implementó una metodología mixta. El diseño fue de triangulación con modelo de convergencia, porque se obtuvieron datos diferentes, pero complementarios sobre el tema (Creswell \& Plano-Clark, 2017). 
EFECTO DE UN PROCESO GRUPAL DE MEDITACIÓN SOBRE EL ESTRÉS Y LAS ESTRATEGIAS DE AFRONTAMIENTO

\section{Fase cuantitativa}

\section{Diseño}

El diseño fue experimental de preprueba-posprueba con grupo control de lista de espera. Las personas privadas delibertad fueron asignadas aleatoriamente auno de dosgrupos. Seconformóungrupoqueparticipóen la intervención (experimental) y un grupo que no participó en el proceso, ni en algún otro tratamiento (control).

La variable independiente (proceso grupal de meditación) consistió en una intervención de 10 sesiones impartidas de 10 a.m. a 11:30 a.m. Inicialmente, cada dos semanas se realizaría una sesión; sin embargo, algunas se suspendieron y reprogramaron debido a requisas, asesinato de una persona recluida y días festivos. Estos eventos fueron experimentados tanto por las personas del grupo experimental como las del grupo control, por lo que no se espera que tengan un efecto diferencial sobre los grupos. Cada sesión se dividió en dos fases: una fase educativa de discusión sobre temas vinculados a la meditación y otra fase de práctica meditativa.

En cuanto a la variable dependiente, se determinaron los niveles de estrés percibido con base en autorreportes sobre las reacciones de estrés a nivel neurovegetativo, motor y cognitivo mediante su evaluación con el Cuestionario de Estrés de Solano-Beauregard (Bertoglia, 1997). La hipótesis de investigación fue que el proceso grupal de meditación disminuye los niveles de estrés en estas tres áreas: motora, neurovegetativa y cognitiva.

\section{Participantes}

La estrategia de muestreo fue no probabilística, por conveniencia. Los participantes se seleccionaron de una lista de personas interesadas en el proceso. Para determinar el tamaño de muestra se utilizó el programa $\mathrm{G}^{*}$ Power, con el análisis prospectivo de poder para un tamaño del efecto de .25 , alfa de .05 , y beta de .80, con dos grupos y dos mediciones, con lo que se estimó un tamaño muestral de 34 .

Los criterios de inclusión fueron los siguientes: estar alfabetizado, no finalizar la sentencia durante la investigación, no estar participando en procesos con objetivos similares y no tener una patología mental severa o consumo excesivo de drogas. Para verificar el cumplimiento de los criterios, se realizó una entrevista con cada persona de la lista. Posteriormente, se efectuó la asignación aleatoria a las condiciones del experimento.

Se inició con un total de 41 personas privadas de libertad. Sin embargo, de las personas del grupo experimental, 5 personas desertaron del proceso; mientras que, de las personas del grupo control, 4 personas se negaron a contribuir en la posprueba. Por ello, la muestra final fue de 32 personas (16 en cada grupo). La edad de los participantes se encontró entre 26 y 58 años $(M=39.09$ años, $D E=9.68)$. La mayoría tenía baja escolaridad, un $78.20 \%$ no concluyó la secundaria. En relación con los años de sentencia, los participantes reportaron cifras entre 10 y 154 años $(M=32.00, D E=24.55)$ y el tiempo de estar en la cárcel osciló entre 2.04 y 21 años $(M=8.36, D E=5.23)$.

\section{Instrumentos con los que se recolectó la información}

Cuestionario de datos de identificación: Para caracterizar a la muestra (edad, escolaridad, etc.), se aplicó un cuestionario impreso. Estos datos se incluyeron posteriormente en los análisis para controlar explicaciones alternativas. 
Cuestionario de Estrés de Solano-Beauregard (Bertoglia, 1997): Es un instrumento que contiene 60 ítems para autovalorar signos de estrés de una forma dicotómica en tres áreas: motora, cognitiva y neurovegetativa. Además, permite extraer el índice promedio general de estrés. Este cuestionario se adaptó a la población penal a través de cuatro entrevistas cognitivas de sondeo concurrente (Smith y Molina, 2011) realizadas a personas recluidas en el CAI JAMC que no fueron parte del experimento. Como resultado, se incluyeron modificaciones al instrumento.

El cuestionario adaptado se redujo a 57 ítems y la escala dicotómica se cambió a una de escala tipo Likert de 5 puntos. También, se modificó la redacción de algunos reactivos. A continuación, se muestran ejemplos de los cambios en algunos ítems. El ítem 28: "Le brincan los músculos del pecho" cambió a "En el último mes, he sentido que me brincan los músculos del pecho sin que se deba a estar haciendo ejercicio". El ítem 51: "Le cuesta concentrarse en lo que hace" se sustituyó por "En el último mes, me ha costado concentrarme en mis actividades diurnas sin que se deba a las interrupciones de otros".

Posteriormente, se estimaron las propiedades psicométricas del instrumento adaptado. Para ello, se aplicó el cuestionario a 139 hombres recluidos en la Unidad de Atención Integral Reynaldo Villalobos que no fueron parte del experimento. Los análisis se efectuaron en el programa estadístico R versión 3.5.1. Se utilizaron los paquetes Psych versión 1.8.10, MBESS versión 4.6.0, MVN versión 5.8 y Lavaan versión 0.6-3.

Como resultado, se obtuvieron buenos indicadores de consistencia interna (escala completa $\alpha$ $=.96$, subescala motora $\alpha=.91$, subescala cognitiva $\alpha=.93$ y subescala neurovegetativa $\alpha=.86$; los valores del omega de McDonald para la escala completa y subescalas no difirieron de los anteriores). Además, la correlación entre las subescalas fue motora y cognitiva de .85; motora y neurovegetativa de .84; cognitiva y neurovegetativa de .77. Finalmente, la correlación entre la escala total con la subescala motora fue de .96 , con la cognitiva de .93 y con la neurovegetativa de .92 .

Adicionalmente, se efectuó un análisis factorial confirmatorio de la escala con el paquete lavaan versión 0.6-3. Se siguieron las recomendaciones de Lei y Wu (2012). Para el manejo de los valores perdidos, se utilizó el estimador de máxima verosimilitud con información completa (FIML, por sus siglas en inglés). Además, debido a que las pruebas de normalidad multivariada resultaron significativas (asimetría $=452.80, p<.001$; curtosis $=9.80, p<.001$ ), se utilizó máxima verosimilitud robusta para la estimación de los parámetros.

También, de acuerdo con Hooper, Coughlan y Mullen (2008), una razón $\chi^{2} / \mathrm{df}$ menor a 5, un CFI (Bentler's Comparative Fit Index) y un TLI (Tucker-Lewis Index) / NNFI (Bentler-Bonett Nonnormed Fit Index) superior a .95 y un RMSEA (Root Mean Square Error of Approximation) en el rango de .05 a .010 son indicadores de un buen ajuste. En el caso del AIC (Akaike's Information Criterion) y el BIC (Bayesian Information Criterion), un valor menor es indicativo de un mejor ajuste. De esta manera, debido a que el modelo no presentó un buen ajuste al utilizar todos los ítems $\left(\chi_{(1536, n=139)}^{2}=3003.804, p\right.$ $<.001 ;$ RMSEA $=.083(.079, .087) ; \mathrm{CFI}=.652 ; \mathrm{TLI} / \mathrm{NNFI}=.639 ; \mathrm{AIC}=22538.126 ; \mathrm{BIC}=22498.229)$, se decidió crear parcelas mediante las cargas factoriales de los ítems. Para ello, se usó el promedio de los ítems, agrupados como se muestra en la tabla 1. 
EFECTO DE UN PROCESO GRUPAL DE MEDITACIÓN SOBRE EL ESTRÉS Y LAS ESTRATEGIAS DE AFRONTAMIENTO

Tabla 1

Parcelas generadas a partir de las cargas factoriales de los items

\begin{tabular}{lll}
\hline Subescala & Parcela & \multicolumn{1}{c}{ Ítems } \\
\hline Síntomas motores & Motor 1 & Ítem 60, Ítem 44, Ítem 38, Ítem 23, Ítem 59, Ítem 06, Ítem 28 \\
& Motor 2 & Ítem 52, Ítem 30, Ítem 13, Ítem 01, Ítem 11, Ítem 22 \\
& Motor 3 & Ítem 58, Ítem 03, Ítem 50, Ítem 07, Ítem 55, Ítem 42 \\
Síntomas cognitivos & Cognitivo 1 & Ítem 40, Ítem 09, Ítem 51, Ítem 56, Ítem 32, Ítem 12, Ítem 53 \\
& Cognitivo 2 & Ítem 47, Ítem 08, Ítem 43, Ítem 27, Ítem 19, Ítem 14 \\
& Cognitivo 3 & Ítem 49, Ítem 37, Ítem 33, Ítem 20, Ítem 48, Ítem 17 \\
Síntomas & Neurovegetativo 1 & Ítem 45, Ítem 57, Ítem 35, Ítem 31, Ítem 10, Ítem 41, Ítem 21 \\
neurovegetativos & Neurovegetativo 2 & Ítem 46, Ítem 34, Ítem 39, Ítem 36, Ítem 29, Ítem 54 \\
& Neurovegetativo 3 & Ítem 16, Ítem 25, Ítem 18, Ítem 04, Ítem 24, Ítem 02 \\
\hline
\end{tabular}

Para este modelo, todas las cargas factoriales y pesos de regresión fueron significativos (ver figura 1). Asimismo, el modelo presentó un buen ajuste $\left(\chi_{(24, n=139)}^{2}=39.921, p=.022 ; R M S E A=.074(.029\right.$, $.114) ; \mathrm{CFI}=.984 ; \mathrm{TLI} / \mathrm{NNFI}=.976 ; \mathrm{AIC}=1748.016 ; \mathrm{BIC}=1741.137)$.

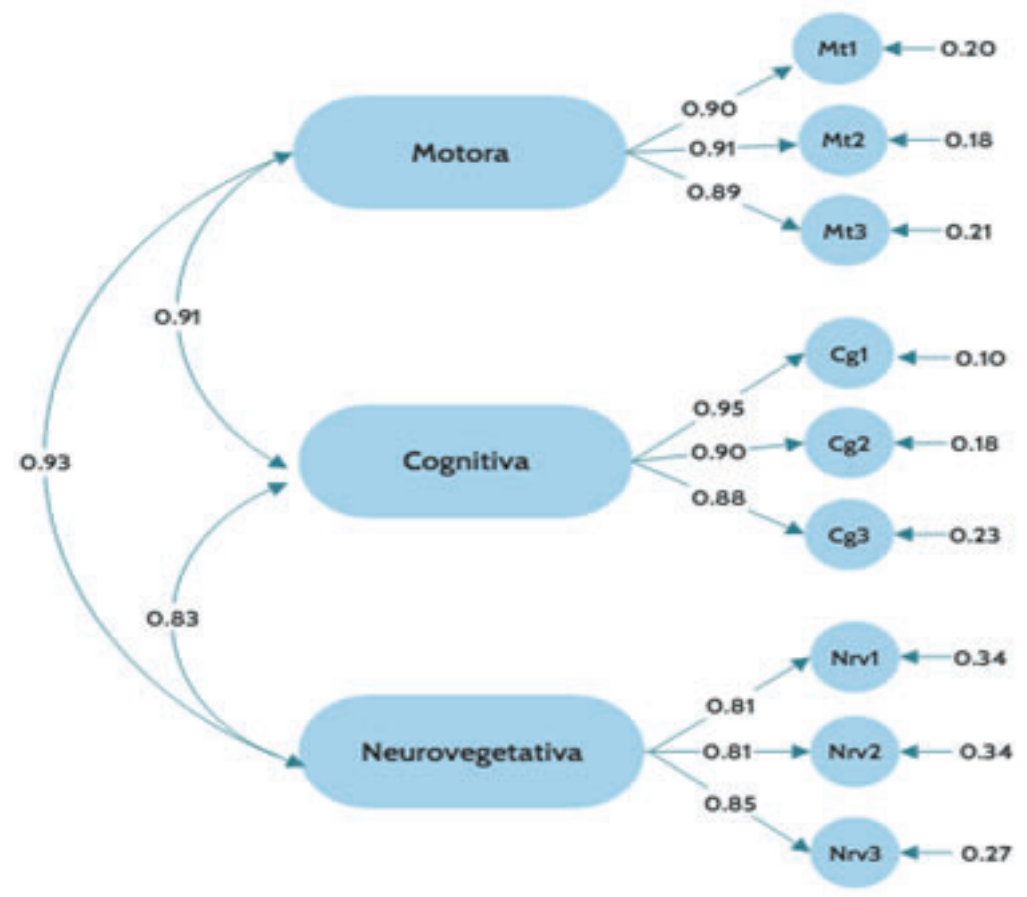

Figura 1. Cargas factoriales y pesos de regresión para la adaptación del Cuestionario de Estrés de SolanoBeauregard (1991). 


\section{Proceso}

Antes de iniciar la investigación, los participantes firmaron un consentimiento informado en el que se les indicó, de forma clara y completa, el tipo de estudio por realizar, período de aplicación, objetivos, duración, alcances e importancia de los resultados esperados. Asimismo, se les informó sobre sus derechos: participación voluntaria, poder retirarse en cualquier momento, derecho a una respuesta clara y satisfactoria sobre las preguntas que surjan durante la investigación y ser notificados de cualquier cambio. También, se informó de las precauciones tomadas para proteger y mantener su confidencialidad. Para resguardar su identidad, se cambiaron los nombres por seudónimos, las grabaciones fueron manipuladas únicamente por el equipo investigador y se eliminaron al finalizar el análisis.

Posteriormente, se aplicaron los instrumentos a los grupos en dos momentos. Antes de iniciar la intervención, se aplicó el cuestionario de datos demográficos y el cuestionario de estrés adaptado. Al finalizar el proceso se administró, nuevamente, el cuestionario de estrés adaptado.

\section{Análisis}

Con los datos de los instrumentos se generó un archivo .csv (comma-separated values) en el programa Excel, que se importó al programa $\mathrm{R}$ versión 3.5.1. Se efectuaron análisis descriptivos de los datos de identificación y del cuestionario de estrés. Seguidamente, se realizaron análisis de varianza (ANOVA) con el fin de poner a prueba las hipótesis estadísticas, así como análisis de covarianza (ANCOVA) para disminuir la posibilidad de explicaciones alternativas. Las covariables fueron las siguientes: nivel de escolaridad, edad, años de sentencia y tiempo en prisión.

\section{Fase cualitativa}

Diseño

Se utilizó un diseño de estudio de casos múltiples para lograr una comprensión exhaustiva dentro de un contexto real y generar una interpretación colectiva (Creswell, 2014; Yin, 2013) sobre los estresores del entorno carcelario y los cambios en las estrategias de afrontamiento asociados a la intervención.

\section{Participantes}

Se utilizó un procedimiento de muestreo intencional completo y homogéneo (Flick, 2015; Izcara, 2014). Se trabajó con los 16 participantes del proceso grupal de meditación; es decir, los hombres que fueron asignados a la condición experimental en la fase cuantitativa. Estas personas tenían entre 26 años y 55 años $\left(M=41.06\right.$ años, $\left.D E=10.26, M_{e}=42, M_{o}=52\right)$. En cuanto al nivel académico, nueve no terminaron la primaria o llegaron hasta sexto grado, cinco cursaron secundaria y solo dos reportaron estudios superiores inconclusos. En relación con los años de sentencia, el promedio fue de 36.37 años $\left(D E=32.95, M_{e}=30, M_{o}=30\right)$ y el promedio de tiempo de estar en prisión fue de 6.97 años $(D E=4.59$, $\left.M_{e}=6, M_{o}=3\right)$.

\section{Técnicas de recolección de información}

Entrevista semiestructurada: Se utilizó un protocolo de entrevista con preguntas que buscaron introducir al entrevistado en áreas temáticas, pero con flexibilidad para adaptarse al discurso de la persona 
EFECTO DE UN PROCESO GRUPAL DE MEDITACIÓN SOBRE EL ESTRÉS Y LAS ESTRATEGIAS DE AFRONTAMIENTO

entrevistada y captar sus puntos de vista (O’Reilly \& Dogra, 2017). Se entrevistó al total de participantes y se grabó cada entrevista para tener un registro preciso.

Observaciones no participantes: En esta observación, el investigador no tiene familiaridad con lo acontecido, en tanto mantiene el rol de agente externo e intenta documentar detalladamente los eventos del entorno (Fàbregues \& Paré, 2016). Se contó con una guía de observación elaborada a partir de los objetivos del estudio para documentar las sesiones del programa de meditación.

\section{Proceso}

Dos personas del equipo de investigación realizaron observaciones de las sesiones del programa de meditación y cada una elaboró su propia bitácora de sesión. Se realizó triangulación de investigadores, al discutir y contrastar estas bitácoras para generar un producto por sesión. Además, se grabaron y se transcribieron las entrevistas para evitar sesgos por atención selectiva.

Cada investigador realizó la codificación de las entrevistas y de los productos finales de las observaciones en el programa Atlas.ti version 7.5.4. Las categorías preestablecidas fueron las siguientes: estresores ambientales (hacinamiento, territorialidad, temperatura, etc.) y afrontamiento (autocontrol, distanciamiento, confrontación, etc.). También, se incluyeron categorías emergentes (estresores: diversidad, problemas de higiene, etc.; afrontamiento: apoyo espiritual). Posteriormente, se realizó una triangulación de investigadores al enlazar las bases de datos y detectar las coincidencias en la codificación. Además, se triangularon los métodos al comparar los productos de las observaciones con las entrevistas efectuadas a los participantes.

\section{Análisis}

Se realizó análisis de contenido con la ayuda de la herramienta de consulta del Atlas.ti para extraer la cantidad de citas por categoría y citas de cada categoría. También, se efectuaron análisis de co-ocurrencia para establecer relaciones e identificar categorías centrales.

\section{Fase de valoración del proceso de meditación}

\section{Diseño}

El diseño fue de teoría fundamentada, porque en el país hay pocos referentes teóricos e investigaciones sobre intervenciones basadas en meditación. Como el interés en esta fase fue contar con premisas sobre el diseño de la intervención, se buscó que de los datos emergiera la teoría, por lo que se utilizó el sistema inductivo para la extracción de categorías (Sim, 2018).

\section{Participantes}

Se utilizó un muestreo intencional (Flick, 2015; Izcara, 2014), porque se seleccionó a los 16 usuarios de la intervención para obtener una valoración del proceso. Además, se buscó a dos personas de la comunidad científica con formación en Psicología y capacitación en meditación para la valoración del diseño, los contenidos y las consignas del proceso grupal de meditación.

Técnicas de recolección de información

Entrevista semiestructurada: En las entrevistas a los participantes de la intervención, se introdujeron algunas preguntas para conocer las valoraciones de ellos sobre las fases del proceso. 
Observaciones no participantes. Con las observaciones, también se valoró el proceso grupal y con las bitácoras trianguladas se generó un documento que sintetiza y describe la intervención.

Entrevista a expertos. Consiste en efectuar una serie de preguntas a expertos en la temática. Ello demanda un juicio fundamentado. La persona entrevistadora se familiariza con el sistema profesional de relevancia de los expertos (Littig \& Pöchhacker, 2014), lo que se hizo a través de la revisión documental y las observaciones no participantes.

Proceso

A los expertos se les entregó anticipadamente la sistematización de la intervención y después se aplicaron las entrevistas, las cuales se transcribieron y procesaron, junto con las entrevistas de los participantes, en el programa Atlas.ti versión 7.5.4. Se codificaron incidentes que se compararon y analizaron para generar las categorías (Belgrave \& Seide, 2019).

\section{Análisis}

Se efectuaron análisis de concurrencia en el Atlas.ti, para identificar categorías centrales. Los resultados se contrastaron con teoría de Psicología de la salud.

\section{Integración de resultados cuantitativos y cualitativos}

Se desarrolló una triangulación de métodos al contrastar los cambios de estrés reportados en la preprueba y la posprueba del grupo experimental con las narraciones de los participantes, que evidenciaban cambios en las reacciones de estrés a partir de la utilización de estrategias de afrontamiento diferentes a las habituales en el contexto, lo que se atribuyó a los aprendizajes del proceso grupal de meditación.

\section{Resultados}

\section{Cuantitativos}

Para mejorar la redacción, cambiar por:

Como se observa en la tabla 2, el análisis de varianza factorial (ANOVA) y el modelo lineal general evidenciaron que no hubo diferencias significativas en los niveles de estrés entre el grupo control y el grupo experimental cuando se utiliza como criterio el puntaje total en la escala $\left(F_{(1,30)}=2.82, p=.10\right)$. Sin embargo, la diferencia sí fue significativa en lo que respecta a la subescala cognitiva $\left(F_{(1,30)}=5.00\right.$, $p=.03)$. Por su parte, al comparar el pretest y el postest, el grupo control no reportó diferencias consistentes ni significativas, mientras que el grupo experimental sí mostró consistentemente disminuciones de los niveles de estrés, los cuales resultaron significativos en el caso de la subescala cognitiva $\left(F_{(1,15)}\right.$ $=5.01, p=.04)$. 
EFECTO DE UN PROCESO GRUPAL DE MEDITACIÓN SOBRE EL ESTRÉS Y LAS ESTRATEGIAS DE AFRONTAMIENTO

Tabla 2

Estadísticos descriptivos y análisis de varianza factorial mixto

\begin{tabular}{|c|c|c|c|c|c|c|c|c|c|}
\hline & \multirow[b]{2}{*}{ Condición } & \multicolumn{2}{|r|}{ Pretest } & \multicolumn{2}{|r|}{ Postest } & \multicolumn{4}{|c|}{ Contraste } \\
\hline & & $N$ & $M(D E)$ & $N$ & $M(D E)$ & $F$ & $g l_{1}$ & $g l_{2}$ & $p$ \\
\hline \multirow[t]{4}{*}{ Total } & Experimental & 16 & $111.38(46.31)$ & 16 & $93.81(32.35)$ & 3.86 & 1 & 15 & .07 \\
\hline & Control & 16 & $126.5(41.14)$ & 16 & $126.31(41.08)$ & 0.00 & 1 & 15 & .97 \\
\hline & Ambas & 32 & $118.94(43.77)$ & 32 & $110.06(39.94)$ & 2.95 & 1 & 30 & .10 \\
\hline & Modelo general & & & & & 2.82 & 1 & 30 & .10 \\
\hline \multirow[t]{4}{*}{ Motor } & Experimental & 16 & $36.38(14.75)$ & 16 & $30.44(11.95)$ & 3.85 & 1 & 15 & .07 \\
\hline & Control & 16 & $42.12(14.59)$ & 16 & $40(12.39)$ & 0.80 & 1 & 15 & .39 \\
\hline & Ambas & 32 & $39.25(14.72)$ & 32 & $35.22(12.92)$ & 3.72 & 1 & 30 & .09 \\
\hline & Modelo general & & & & & 0.98 & 1 & 30 & .33 \\
\hline \multirow[t]{4}{*}{ Cognitivo } & Experimental & 16 & $39.25(17.14)$ & 16 & $31.81(11.48)$ & 5.01 & 1 & 15 & .04 \\
\hline & Control & 16 & $43.81(15.42)$ & 16 & $45.25(15.94)$ & 0.44 & 1 & 15 & .52 \\
\hline & Ambas & 32 & $41.53(16.2)$ & 32 & $38.53(15.27)$ & 2.23 & 1 & 30 & .15 \\
\hline & Modelo general & & & & & 5.00 & 1 & 30 & .03 \\
\hline \multirow[t]{4}{*}{ Neurovegetativo } & Experimental & 16 & $35.75(15.67)$ & 16 & $31.56(10.7)$ & 1.97 & 1 & 15 & .18 \\
\hline & Control & 16 & $40.56(13.5)$ & 16 & $41.06(14.66)$ & 0.07 & 1 & 15 & .79 \\
\hline & Ambas & 32 & $38.16(14.59)$ & 32 & $36.31(13.52)$ & 1.85 & 1 & 30 & .13 \\
\hline & Modelo general & & & & & 1.79 & 1 & 30 & .19 \\
\hline
\end{tabular}

Además, con el análisis de covarianza (ANCOVA) se determinó que las variaciones no se asociaron a otras variables como edad, escolaridad, años de sentencia o tiempo encarcelado.

\section{Cualitativos}

Para contextualizar, se indagaron los estresores ambientales que percibieron las personas participantes y requieren afrontamiento. En primer lugar, destacó que los usuarios indicaron que el ambiente carcelario es un entorno de alto riesgo, que demanda esfuerzos constantes para sobrevivir. Así, al referirse al encarcelamiento Julio expresó:

Una experiencia difícil, sufrida, riesgosa. La persona común no sabe la forma en que se vive en este ambiente, es sumamente peligroso. Hoy nosotros estamos vivos, mañana no sabemos si vamos a amanecer. En realidad, aquí no se vive, aquí lo que se hace es que se sobrevive.

En cuanto a los estresores, se evidenció que la sobrepoblación carcelaria genera hacinamiento, lo cual juega un papel importante en la forma en que se dan los otros estresores. Por ejemplo, la cantidad 
excesiva de personas conlleva a la convivencia con gente diversa y se generan conflictos, tal como se denota en el siguiente relato: ... porque, diay, mucho hacinamiento, hay personas que piensan diferente a las demás, entonces algún compañero por alguna cosita ya se va a sentir ofendido y van a pasar problemas y todo eso (Fernando $\mathrm{S}$ ).

Otros estresores que sobresalieron fueron el control ambiental para satisfacer necesidades y los problemas de territorialidad por la posesión, la apropiación y la defensa del espacio. Al respecto, debido a las limitaciones de personal institucional, a lo interno de los pabellones ${ }^{1}$, se instauran regímenes ${ }^{2}$ y se dan luchas por territorialidad, lo que impacta directamente sobre aspectos como la llamada diaria que, a veces, es obstaculizada porque las personas del régimen acaparan el teléfono. El siguiente relato revela esta situación: dentro del pabellón se maneja un régimen, donde hay alguien que gobierna que tiene a sus... aqui le llaman perros. Ellos tratan de mantener un orden y entonces entre ellos mismos se va a ver esos favoritismos (Lucas).

También, el derecho a la atención médica oportuna se afecta por dinámicas de control ambiental: ...un muchacho se cayó de la cama y se le partió la muñeca totalmente (...) tuvimos que irnos todos a hacer presión ahi para que lo sacaran. Duró como unos 45 minutos con la mano quebrada ahí en el portón, esperando que llegara el oficial a sacarlo (Eliomar).

La infraestructura inadecuada es otro factor relevante del ambiente, porque, debido a la antigüedad y el deterioro, el edificio no reúne las condiciones para albergar dignamente a la población creciente. Esto influye negativamente sobre aspectos fundamentales como la higiene y podría aumentar la necesidad de requerir atención médica. En palabras de ellos: digamos aqui hay tantas suciedades, sinceramente, que hace poco le cortaron el pie a un pillo por ahi, por la misma vara y un chorro para que lo atiendan los médicos también (Norman).

La inadecuación estructural también conlleva problemas de temperatura, olores desagradables y exceso de ruido. Los siguientes fragmentos permiten apreciar la magnitud de estos estresores:

Bueno, ahorita adentro es un horno porque como es un cuadrito, el calor que hay adentro se concentra, entonces corre hacia los cuartos, entonces es muy sofocado (Leónidas).

Estar en el pabellón donde nosotros estábamos es como que usted está las 24 horas, bueno, no, por lo menos de las 6 de la mañana a las 10 de la noche en el centro del mercado, o sea siempre hay bulla, alguien grita, el tele prendido duro (Walter).

Los relatos anteriores dejan en evidencia lo complejo del ambiente, el cual requiere una serie de estrategias para afrontar las situaciones que se presentan.

Vinculado con lo anterior, en la figura 2, que muestra la cantidad de personas por estilo de afrontamiento previo y posterior al proceso, se observa que gran parte de los usuarios reconocieron que, antes de participar en la intervención, usualmente actuaban de forma confrontativa, porque sus conductas

\footnotetext{
${ }^{1}$ Pabellones: Divisiones estructurales dentro del Ámbito C, donde se distribuyen las personas privadas de libertad.

${ }^{2}$ Regímenes: estructuras y dinámicas de poder dentro de cada pabellón al mando de un líder apodado "jachudo" y sus colaboradores llamados "perros".
} 


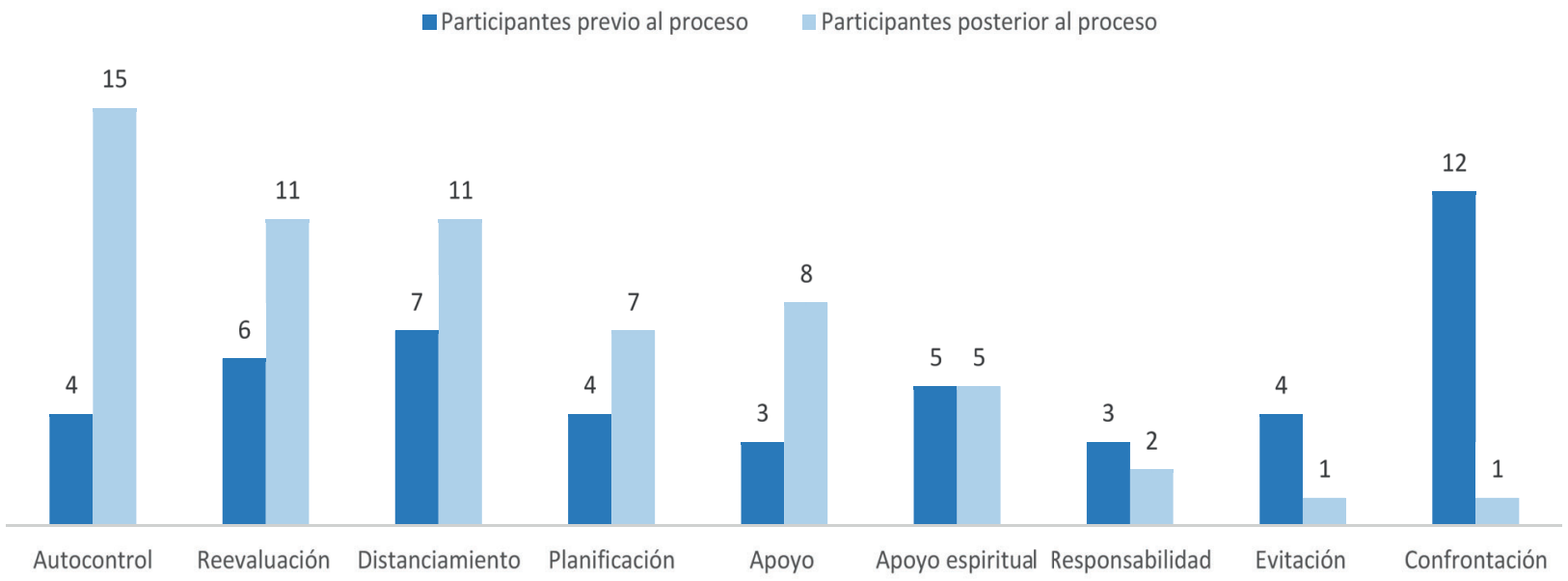

Figura 2. Cantidad de personas por estilo de afrontamiento, previo y posterior al proceso.

eran directas, hostiles y arriesgadas. Ellos indicaron que las condiciones del entorno les provocaban estar a la defensiva.

Una cita, que refleja la forma confrontativa de actuar en ese entorno frente a los problemas, se presenta a continuación:

Antes, andaba con el carácter alzado, andaba más a la defensiva, eh... son cosas con las que uno convive aqui, para nadie es un secreto, andaba hasta con fierro. Uno andaba ahi, como le digo, a la defensiva (Eliomar).

Sin embargo, casi todos los participantes expresaron que el proceso les ayudó a cambiar sus formas de afrontamiento, porque les enseñó a utilizar la meditación para tener control sobre sí mismos y no dejarse determinar por factores externos. Con ello, lograron regular sus respuestas emocionales y acciones, lo que se aprecia en la figura 2, específicamente en la cantidad de personas que dijo utilizar el autocontrol gracias al proceso grupal de meditación. A continuación, se presentan relatos de los usuarios en los que se evidencian las enseñanzas de la intervención:

Aprendes a tomar decisiones, a tener un mejor punto de vista de las cosas, a buscarle la solución, no hacer el problema más grande, sino inyectarle a la mente lo que has aprendido y aprender a tener autocontrol, no dejarse explotar por esas situaciones (Eliomar).

... pensar las cosas antes de actuar (...) antes era uno muy impulsivo, ahora uno trata de estudiar los problemas y cómo resolverlos sin utilizar la violencia (Alberto).

Además, varios participantes indicaron que la intervención les ayudó a acudir a estrategias como el distanciamiento para separarse de las situaciones problemáticas. Al respecto, cabe resaltar que algunos refirieron al distanciamiento en un plano cognitivo para no pensar en el problema y evitar que les afecte. A continuación, se presentan dos citas que denotan lo indicado: 
Diay, antes era como más escandaloso uno, diay, se ponía a decirle: — ¡cállate! - o así, entonces, ya uno lo que hace es mejor apartarse y andar en otro rumbo (Javier).

El hecho de poder desconectarse un poco de ese tipo de ambiente, de esa presión, que uno sabe que hay gente que anda con cuchillos. Poder tranquilizarse uno mismo, desconectarse, por decirlo así, de esas situaciones, de ese ambiente es de mucha ayuda (Walter).

También, relataron que el proceso les ayudó a analizar las situaciones para buscar soluciones (planificación). Asimismo, mencionaron que la intervención les hizo ver que podían crear un significado positivo ante ciertas experiencias para el crecimiento personal (reevaluación):

[Refiriéndose a la meditación]. Es que uno tiende, cómo le digo, tiende a meterse a lo interno, hacia uno mismo, a pensar y ver qué sirve y qué no sirve, porque si va a tomar una decisión que no le sirve a uno para qué (Evelio).

En síntesis, de acuerdo con la opinión de los participantes, el proceso grupal de meditación promovió un cambio positivo, porque pasaron de comportamientos confrontativos a estados de autocontrol, que posibilitaron tomar distancia de las situaciones problemáticas, reevaluar los acontecimientos estresantes y planificar formas más adecuadas de actuar.

\section{Valoración del proceso}

Con la valoración del proceso grupal de meditación, se identificaron fortalezas y aspectos por mejorar. Así, se visualizaron como aspectos positivos la empatía del equipo facilitador, el uso de referentes fisiológicos para explicar efectos de la meditación, la ejemplificación con situaciones del contexto y la cohesión grupal que se logró. Con respecto a la dinámica grupal, se recomendó reforzarla desde el inicio con actividades que fomenten más la unidad.

Asimismo, se recomendó evitar informar demasiado sobre los beneficios de la meditación para evitar expectativas muy altas y sentimientos de frustración ante sensaciones displacenteras. Es importante validar cualquier experiencia de los participantes. También, se recomendó cambiar la palabra "relajación" por directrices operativas durante la consigna.

En cuanto a la frecuencia y el horario, se consideró apropiado realizar una sesión por semana de forma consecutiva y programar las sesiones más temprano o después del almuerzo. También, se recomendó programar las sesiones mediante la intercalación de la práctica de meditación con la indagación de la experiencia e incluir más técnicas que dinamicen y aumenten la participación de los usuarios.

Se sugirió ir aumentando el tiempo de la meditación, así como fomentar la adhesión a la práctica con material de apoyo y la asignación de tareas. En esta línea, los expertos indicaron que meditar es lo más importante, por lo que sugirieron focalizar menos en los contenidos y más en el ejercicio e indagación de la experiencia de los participantes.

Finalmente, los expertos resaltaron que el proceso es idóneo por ser un recurso de uso personal, gratuito y de fácil acceso, necesario para las personas recluidas dadas las condiciones de la cárcel. Sin embargo, la intervención puede rediseñarse para maximizar los beneficios. 
EFECTO DE UN PROCESO GRUPAL DE MEDITACIÓN SOBRE EL ESTRÉS Y LAS ESTRATEGIAS DE AFRONTAMIENTO

\section{Discusión}

En esta investigación se buscó conocer el efecto de un proceso grupal de meditación sobre el estrés y las estrategias de afrontamiento de un grupo de 16 hombres privados de libertad en el CAI JAMC. Se implementó una metodología mixta con una fase cuantitativa que dio cuenta de los cambios en los niveles de estrés y una fase cualitativa para explorar las estrategias de afrontamiento en ese contexto particular. Además, se realizó una valoración del diseño, los contenidos y las consignas de la intervención para evaluar su idoneidad.

En cuanto a los resultados cuantitativos, estos se encuentran en la línea de estudios como los de Auty, Cope y Liebling (2017), Barnert et al. (2014), Le y Proulx (2015), Nidich et al. (2016), OrmeJohnson y Barnes (2014) y Yoon, Slade y Fazel (2017), quienes indican que la terapia cognitivo-conductual y la meditación tiene efectos positivos en las personas recluidas. Cabe destacar que la intervención del CAI JAMC integraba la práctica de meditación con una fase psicoeducativa que contenía elementos de terapia cognitiva-conductual. En este contexto, sobresale la reducción significativa de las reacciones de estrés en la subescala cognitiva dentro del grupo experimental. Esos efectos podrían tener relación con el énfasis que se le dio a la meditación como recurso para desarrollar la capacidad de observar los acontecimientos de la vida cotidiana desde la calma, lo que posibilita la identificación y la regulación de los pensamientos, las respuestas emocionales y los comportamientos impulsivos (Audy et al., 2017; Creswell, 2017).

El equipo investigador considera importante consignar que la práctica de meditación desarrollada en el CAI JAMC se fundamenta en la meditación Vipassana, por focalizarse en la respiración y el silencio. Sin embargo, ese tipo de meditación es difícil de implementar en cárceles costarricenses, porque implica un horario estricto de 10 horas de práctica meditativa durante 10 días de retiro, en los cuales se evita la comunicación con otras personas participantes y hasta el décimo día se posibilita la interacción (Audy et al., 2017). Con base en lo anterior, se considera que el ejercicio implementado podría ser una categoría heterogénea, porque utiliza principios de la meditación Vipassana, pero con una estructura diferente al desarrollar las sesiones con un componente educativo, una vez a la semana o cada quince días.

Un aspecto que pudo incidir en los resultados fue la extensión de la fase psicoeducativa en cada sesión, puesto que generó que se dedicara menos tiempo para practicar meditación (alrededor de 12 minutos por sesión). Esto posiblemente tuvo un efecto en los resultados para las subescalas motora y neurovegetativa; específicamente, puede ser una razón de que no se encontraran diferencias significativas, pues la meditación informa sobre la tensión y la distensión en la musculatura y de otras reacciones fisiológica (Schoeberlein, 2017), por lo que la reducción en el tiempo dedicado a esta práctica puede haber reducido sus potenciales beneficios.

Además, en los resultados cualitativos se evidenció la presencia de múltiples estresores, entre los que destaca el hacinamiento, el control ambiental, la territorialidad y la infraestructura inadecuada. Lo anterior concuerda con lo señalado por diversos organismos sobre la necesidad de realizar mejoras en los centros penitenciarios del país para resguardar los derechos humanos de las personas privadas de libertad (Mecanismo Nacional de Prevención de la Tortura, 2018). 
Al respecto, al contrastar los resultados cualitativos y cuantitativos, se identificó que la carga ambiental del contexto penitenciario pudo influir en que no se obtuviera diferencias significativas en los niveles de estrés entre el grupo control y el grupo experimental cuando se utiliza como criterio el puntaje total en la escala. En ese sentido, es importante recordar que el estrés implica la relación individuo-entorno y aunque esta interacción se modifica cuando la persona utiliza formas de afrontamiento diferentes (De Camargo, 2010; Fernández, 2009; Lazarus \& Folkman, 1986), puede resultar muy difícil reducir de forma significativa las reacciones de estrés en un entorno donde la incidencia del sujeto está mediada por el control institucional y las estructuras de poder dentro de los pabellones.

No obstante, los contenidos de la fase psicoeducativa los invitaron a reconocerse y retomar el control de sus emociones y conductas. Dicho aspecto, según el equipo investigador, pudo generar un impacto positivo en los participantes, porque les posibilitó restablecer el control de sí mismos, puesto que es probable que ellos hayan olvidado su potencial de autorregulación, por la dependencia institucional y las dinámicas de poder a lo interno.

Aunado a lo anterior, durante la práctica meditativa, la concentración en la relajación física, la concentración en la respiración y la concentración en el silencio, integraron un ejercicio donde la persona, de manera intencional y voluntaria, focalizaba la atención en sí misma para su bienestar. Así, según los relatos de la mayoría de los participantes, esta práctica les permitió centrarse en el aquí y ahora para mantenerse en el presente, detener la cadena de pensamientos asociados a la reactividad y desvincularse momentáneamente del ambiente extremo en que se encuentran. Por esos motivos, los participantes refirieron que el proceso grupal de meditación les permitió modificar las formas en que afrontaban la situación carcelaria, porque aprendieron la importancia de no actuar de forma confrontativa. Asimismo, señalaron que el proceso les enseñó a regular sus emociones y a analizar las circunstancias para cambiar sus percepciones.

En este sentido, los participantes comentaron que, a raíz del proceso, acudieron al distanciamiento para evitar problemas y a la planificación para resolverlos. Asimismo, indicaron que mejoraron sus relaciones con otras personas, pues aumentaron la empatía al reevaluar las situaciones desde un estado de tranquilidad, que les permite ponerse en el lugar del otro.

En cuanto al diseño de la intervención, se consideró idóneo, porque es de bajo costo y proporciona elementos teóricos y metodológicos que contribuyen a las personas en ese contexto. Además, las áreas por mejorar en cuanto a la implementación permiten vislumbrar la posibilidad de reestructurar el diseño para maximizar los resultados de próximas intervenciones.

A modo de conclusión, resalta que este fue un estudio pionero sobre meditación con población penal en Costa Rica, el cual evidenció los efectos de la intervención en las reacciones de estrés y las estrategias de afrontamiento. Así, el proceso fue una alternativa ante las condiciones de riesgo de la prisión, mientras se gestan cambios institucionales que requieren análisis contextualizados y voluntad política para que se garantice los derechos de las personas recluidas.

Las limitaciones de la investigación refieren a las características institucionales que, como se mencionó, afectaron el tamaño de la muestra y la frecuencia de las sesiones. Además, existe la posibilidad de que las respuestas de los participantes estuvieran influidas por la deseabilidad social, debido a la 
EFECTO DE UN PROCESO GRUPAL DE MEDITACIÓN SOBRE EL ESTRÉS Y LAS ESTRATEGIAS DE AFRONTAMIENTO

subordinación institucional y a que sus conductas son evaluadas constantemente en las valoraciones de seguimiento. Pese a lo anterior, el equipo investigador considera que la tendencia a respuestas favorables probablemente se redujo, porque los objetivos de la intervención no estaban vinculados con la tipología del delito, lo que contribuyó a una mayor apertura en el espacio.

\section{Reconocimientos}

Deseamos expresar nuestro reconocimiento y gratitud a la psicóloga Kenia Lobo Miranda y al doctor Ignacio Salom Echeverría por la dedicación y el compromiso con el proceso grupal de meditación y el apoyo brindado a la investigación. También, se agradece a Esteban Navarro Díaz, Esteban Carvajal Angulo y Jorge Ernesto Peñaloza Castañeda por las asesorías durante la investigación.

\section{Referencias}

Auty, K. M., Cope, A., \& Liebling, A. (2017). A systematic review and meta-analysis of yoga and mindfulness meditation in prison: Effects on psychological well-being and behavioural functioning. International Journal of Offender Therapy and Comparative Criminology, 61(6), 689-710. https://doi. org/10.1177/0306624X15602514

Barnert, E. S., Himelstein, S., Herbert, S., Garcia-Romeu, A., \& Chamberlain, L. J. (2014). Exploring an intensive meditation intervention for incarcerated youth. Child \& Adolescent Mental Health, 19(1), 69-73. https://doi. org/10.1111/camh.12019

Belgrave, L., \& Seide, K. (2019). Coding for grounded theory. En A. Bryant \& K. Charmaz (Eds.), The SAGE handbook of current developments in grounded theory (pp. 167-185). Londres: SAGE.

Bertoglia, J.P. (1997). Adaptación del "Cuestionario sobre estrés SolanoBeauregard" para la medición del nivel de estrés en el personal de oficinas centrales y periféricas del Banco Nacional de Costa Rica. (Tesis de licenciatura). Universidad de Costa Rica, Costa Rica.

Cólica, P.R. (2015). Estrés manual diagnóstico: La explicación psicobiológica de los síntomas. Buenos Aires, Argentina: Brujas.

Cozzo, G., \& Reich, M. (2016). Estrés percibido y calidad de vida relacionada con la salud en personal sanitario asistencial. PSIENCIA. Revista Latinoamericana de Ciencia Psicológica, 8(1), 1-15.

Creswell, J. (2014). Research design: Qualitative, quantitative, and mixed methods approaches. California: SAGE.

Creswell, J. (2017). Mindfulness interventions. Annual Review of Psychology, 68, 491-516.

Creswell, J. W., \& Plano-Clark, V. L. (2017). Designing and conducting mixed methods research (3a ed.). Los Ángeles: SAGE

De Camargo, B. (2010). Estrés, síndrome general de adaptación o reacción general de alarma. Revista Médico Cientifica, 17(2), 78-86. Recuperado de http://www.revistamedicocientifica.org/index.php/rmc/article/ view/103/637

Fàbregues, S., \& Paré, M. H. (2016). La observación. En F.S. Fàbregues, N.J. Meneses, G.D. Rodríguez, \& M.H. Paré (Eds.), Técnicas de investigación social y educativa (pp. 120-138). Barcelona: Editorial UOC. 
Fernández, M. (2009). Estrés percibido, estrategias de afrontamiento y sentido de coherencia en estudiantes de enfermería: Su asociación con salud psicológica y estabilidad emocional (Tesis de doctorado). Universidad de León, México.

Flick, U. (2015). El diseño de investigación cualitativa. Madrid: Morata.

García-Jarillo, M., Caravaca-Sánchez, F., Sánchez-Alcaraz, C., \& Luna, A. (2016). Psychosocial stressors perceived in the process of social reintegration and compliance time remaining in prison sentence. Revista Española de Sanidad Penitenciaria, 18(2), 49-95. Recuperado de http://scielo.isciii.es/scielo. php?script=sci_arttext\&pid=S1575-06202016000200003\&lng=es\&tlng=en

Goleman, D. (2003). Emociones destructivas: Cómo comprenderlas y dominarla. Buenos Aires: B Argentina.

González, B., \& Escobar, A. (2002). Neuroanatomía del estrés. Revista Mexicana de Neurociencia, 3(5), 273-282.

Harrison, E. (2004). Meditaciones breves para vivir sin estrés. Barcelona, España: Oniro.

Hooper, D., Coughlan, J., \& Mullen, M. R. (2008). Structural equation modelling: Guidelines for determining model fit. The Electronic Journal of Business Research Methods, 6(1), 53-60. Recuperado de http://www.ejbrm.com/ issue/download.html?idArticle $=183$

Izcara, S. (2014). Manual de investigación cualitativa. México D.F., México: Fontamara.

Lazarus, R., \& Folkman, S. (1986). Estrés y procesos cognitivos. Barcelona: Martínez Roca.

Le, T. N., \& Proulx, J. (2015). Feasibility of mindfulness-based intervention for incarcerated mixed-ethnic Native Hawaiian/Pacific Islander youth. Asian American Journal of Psychology, 6(2), 181. http://dx.doi.org/10.1037/ aap0000019

Lei, P., \& Wu, Q. (2012). Estimation in structural equation modeling. En R.H. Hoyle. (Ed.), Handbook of structural equation modeling (pp. 164-180). Nueva York: Guilford.

Littig, B., \& Pöchhacker, F. (2014). Socio-translational collaboration in qualitative inquiry: The case of expert interviews. Qualitative Inquiry, 20(9), 1085-1095. https://doi.org/10.1177/1077800414543696

Martínez, D. (2014). Análisis de la relevancia de la política pública para la atención de la salud mental en Costa Rica. Enfermería Actual en Costa Rica, 26, 1-8. Recuperado de https://revistas.ucr.ac.cr/index.php/enfermeria/ article/view/13699

Mecanismo Nacional de Prevención de la Tortura (2018). 2017 Informe anual de labores. Recuperado de http:// www.dhr.go.cr/transparencia/participacion_ciudadana/prevencion_de_la tortura/informes/informe_anual mnpt 2017.pdf

Moser, G. (2014). Psicología ambiental: Aspectos de las relaciones individuo-medioambiente. Bogotá: Ecoe Ediciones.

Nidich, S., O’Connor, T., Rutledge, T., Duncan, J., Compton, B., Seng, A., \& Nidich, R. (2016). Reduced trauma symptoms and perceived stress in male prison inmates through the transcendental meditation Program: A Randomized Controlled Trial. Perm J, 20(4), 43-47. https://doi.org/10.7812/TPP/16-007

O'Reilly, M., \& Dogra, N. (2017). Interviewing children and young people for research. Londres: SAGE. 
EFECTO DE UN PROCESO GRUPAL DE MEDITACIÓN SOBRE EL ESTRÉS Y LAS ESTRATEGIAS DE AFRONTAMIENTO

Orme-Johnson, D., \& Barnes, V. (2014). Effects of the transcendental meditation technique on trait anxiety: a metaanalysis of randomized controlled trials. The Journal of Alternative and Complementary Medicine, 20(5), 330341. https://doi.org/10.1089/acm.2013.0204

Orme-Johnson, D. W., \& Moore, R. M. (2003). First prison study using the transcendental meditation program: La Tuna Federal Penitentiary, 1971. Journal of Offender Rehabilitation, 36(1-4), 89-95.

Perelman, A. M., Miller, S. L., Clements, C. B., Rodríguez, A., Allen, K., \& Cavanaugh, R. (2012). Meditation in a deep south prison:Alongitudinal study of the effects of Vipassana. Journal Of Offender Rehabilitation, 51(3), 176-198.

Pham, K. H. (2013). Outcomes of a recreation therapy yoga meditation intervention on prison inmates'spiritual wellbeing (Tesis de maestría). San Jose State University, California.

Rainforth, M. V., Alexander, C. N., \& Cavanaugh, K. L. (2003). Effects of the transcendental meditation program on recidivism among former inmates of folsom prison: Survival analysis of 15 -year follow-up data. Journal Of Offender Rehabilitation, 36(1-4), 181-203.

Ramos, P. (2017). Inteligencia emocional: Control del estrés (3a. ed.). Málaga: ICB S.L.

Ronel, N., Frid, N., \& Timor, U. (2013). The practice of positive criminology: A Vipassana course in prison. International Journal of Offender Therapy and Comparative Criminology, 57(2), 133-153. https://doi. org $/ 10.1177 / 0306624 X 11427664$

Salom, I. (2005). Meditación y bienestar. Geosalud. Medicina Natural. Recuperado de: http://www.geosalud.com/ medicinanatural/meditacion.htm

Sánchez, M. E. (2010). Estrés, un problema psicosocial: La psicología funcional del sí. En M.E. Sánchez \& C. López (Eds.). Estrés y salud: Aportaciones desde la psicología social (pp. 47-65). D.F. México: Editorial Miguel Ángel Porrúa.

Schoeberlein, D. (2017). Mindfulness: Vivir con atención plena en casa, en el trabajo, en el mundo. Bilbao: Desclée De Brouwer.

Sim, J. (2018). Grounded theory. En B. Frey (Ed.), The SAGE encyclopedia of educational research, measurement, and evaluation (pp. 754-756). California: SAGE

Smith, V., \& Molina, M. (2011). Cuaderno Metodológico 5. La entrevista cognitiva: Guía para su aplicación en la evaluación y mejoramiento de instrumentos de papel y lápiz. San José: Instituto de Investigaciones Psicológicas, Universidad de Costa Rica.

Ugarte, A. M. (2010). El impacto psicoambiental: Los problemas ambientales como problemas sociales. En R. Sapiains (Ed.), El dilema del castor (pp. 261-312). Santiago: Universidad de Chile.

Vannoy, S. D., \& Hoyt, W. T. (2004). Evaluation of an anger therapy intervention for incarcerated adult males. Journal of Offender Rehabilitation, 39(2), 39-57.

Weiss, B. (2007). Meditación: Cómo dejar atrás las tensiones y el estrés y alcanzar la paz. Barcelona: Editorial B.

Yin, R. K. (2013). Case study research: Design and methods (5a. ed.). California: SAGE. 
Yoon, I., Slade, K., \& Fazel, S. (2017). Outcomes of psychological therapies for prisoners with mental health problems: A systematic review and meta-analysis. Journal of consulting and clinical psychology, 85(8), 783-802. http://dx.doi.org/10.1037/ccp0000214

Recibido: 31 de julio de 2019 Revisión recibida: 18 de diciembre de 2019

Aceptado: 24 de enero de 2020 
EFECTO DE UN PROCESO GRUPAL DE MEDITACIÓN SOBRE EL ESTRÉS Y LAS ESTRATEGIAS DE AFRONTAMIENTO

\section{Sobre las autoras y los autores:}

María Nazareth Mora-Arias es licenciada en Psicología, egresada de la Licenciatura en Ciencias de la Educación con énfasis en Administración de la Educación No Formal y Bachiller en Enseñanza de la Psicología. Labora como asesora académica del Programa de Posgrado en Especialidades Médicas e investigadora del Instituto de Investigación en Educación, ambas unidades de la Universidad de Costa Rica. Además, trabaja como docente de Investigación Cualitativa.

Lízbeth Solano-Alpízar es licenciada en Psicología de la Universidad de Costa Rica. Desde el 2005, se desempeña como consultora en proyectos con diversas organizaciones públicas y privadas para la promoción de los derechos humanos de poblaciones vulnerabilizadas en Costa Rica.

Bradly Marín-Picado es licenciado en Psicología y máster en Ciencias Cognoscitivas, profesor e investigador de la Universidad de Costa Rica. Funge como docente en la Escuela de Psicología e investigador en el Instituto de Investigaciones Psicológicas. Su trabajo se centra en el uso de metodologías de registro fisiológico para el estudio de procesos cognitivos y emocionales. Cuenta con varias publicaciones a nivel nacional e internacional.

Jorge Esteban Prado-Calderón es licenciado en Psicología y Bachiller en Enseñanza de la Psicología por la Universidad de Costa Rica, así como estudiante de Ciencias Cognoscitivas. Se desempeña como docente en la Escuela de Psicología de la UCR, donde imparte cursos de investigación, historia de la Psicología, y Psicología cognitiva. Asimismo, es investigador y profesor de métodos de investigación y didáctica en el Centro de Capacitación en Educación a Distancia de la Universidad Estatal a Distancia. Cuenta con 18 publicaciones académicas, 26 ponencias, 12 proyectos de investigación y ha participado en comités científicos de simposios y congresos nacionales e internacionales.

Publicado en línea: 15 de abril de 2020 